\title{
AUTONOMOUS RAILING AND TRAPPING OF MICROBEADS FOR CONTINUOUS FLOW MULTI-STAGE MICROFLUIDIC PROCESSES
}

\author{
Ryan D. Sochol ${ }^{l}$, William E. R. Krieger ${ }^{l}$, Luke P. Lee ${ }^{2}$ and Liwei Lin ${ }^{l}$ \\ ${ }^{1}$ Department of Mechanical Engineering, ${ }^{2}$ Department of Bioengineering \\ Berkeley Sensor and Actuator Center, Biomolecular Nanotechnology Center \\ University of California, Berkeley, USA
}

\begin{abstract}
"Multi-stage" fluidic mixing processes are critical to a wide range of chemical and biological assays (e.g., immunoassays). Unfortunately, the majority of biochemical assays suffer from laborious and time-intensive fluidic mixing procedures in which distinct reagents and/or washes are loaded sequentially and separately (i.e., one-at-a-time). Previously, we introduced the first microfluidic railing system capable of hydrodynamically guiding microbeads into discrete, adjacent flow streams in order to autonomously accomplish multi-stage fluidic mixing reactions on-chip. However, microbead immobilization and signal detection were only possible after reaction completion, which poses a significant problem for bead-based assays that require microbead visualization during intermediate phases of multi-stage processes. To overcome this limitation, here we present a single-layer, "continuous flow" microfluidic system that utilizes microposts arrayed in sections at angles of $1^{\circ}, 15^{\circ}$, and $1^{\circ}$ (with respect to the flow direction) to: ( $i$ ) rail suspended microbeads into a trapping area, (ii) trap select numbers of microbeads, and then (iii) transport subsequent microbeads into adjacent flow streams, respectively. By enabling both autonomous bead-based mixing reactions as well as microbead immobilization during each fluidic mixing stage, the presented continuous flow microfluidic "rail-and-trap" system offers a simple, yet powerful methodology for applications in chemical and biological fields, such as molecular diagnostics.
\end{abstract}

\section{KEYWORDS}

Microfluidic Railing, Microbeads, Trapping, Continuous Flow

\section{INTRODUCTION}

Microbead Functionalization for Biochemical Assays

For multi-stage chemical and biological reaction processes that require fluidic mixing with numerous reagents and/or washes, microfluidic systems offer a variety of benefits, such as low reagent volumes and enhanced reaction kinetics [1-3]. Bead-based microfluidic technologies are especially advantageous for accomplishing biochemical assays (i.e., by using microbeads as substrates) due to high surface-to-volume ratios and the ability to mix-and-match microbeads corresponding to specific screenings [4-9]. In particular, microbeads can be functionalized with diverse surface modifications, such as molecular probes capable of detecting DNA analytes and inflammatory cytokines [9-12]. Unfortunately, bead-based microfluidic systems that are employed to perform multi-stage biochemical assays near-universally require external observation and/or regulation during device operation.

\section{Microfluidic Railing for Continuous Flow Mixing Processes}

Recently, microfluidic research has shifted toward systems with autonomous on-chip functionalities [13-15], and microfluidic railing techniques offer powerful means for autonomously transporting microparticulates into discrete, parallel flow streams (i.e., under continuous input flow conditions). Previously, researchers have achieved microfluidic railing for both photopatterned microstructures $[16,17]$ and microdroplets [18-20]. For example, multi-layer photolithographic processes have been used to etch grooves into microchannels that act as tracks in order to rail (i.e., guide) photopolymerized microstructures $[16,17]$ and microdroplets [18]. To bypass the limitations associated with multi-layer fabrication processes (e.g., increased costs, time, and labor), researchers have also developed single-layer microfluidic systems for railing microdroplets using comparatively smaller microposts (alternatively referred to as micropillars) $[19,20]$. Previously, we presented the first microfluidic railing system capable of handling suspended microbeads, which we employed to passively accomplish a 10-stage molecular synthesis process on microbead substrates [21]. Here, we adapt this bead-based railing system to enable microbead immobilization and visualization during each step of multi-stage fluidic mixing processes (Fig. 1).

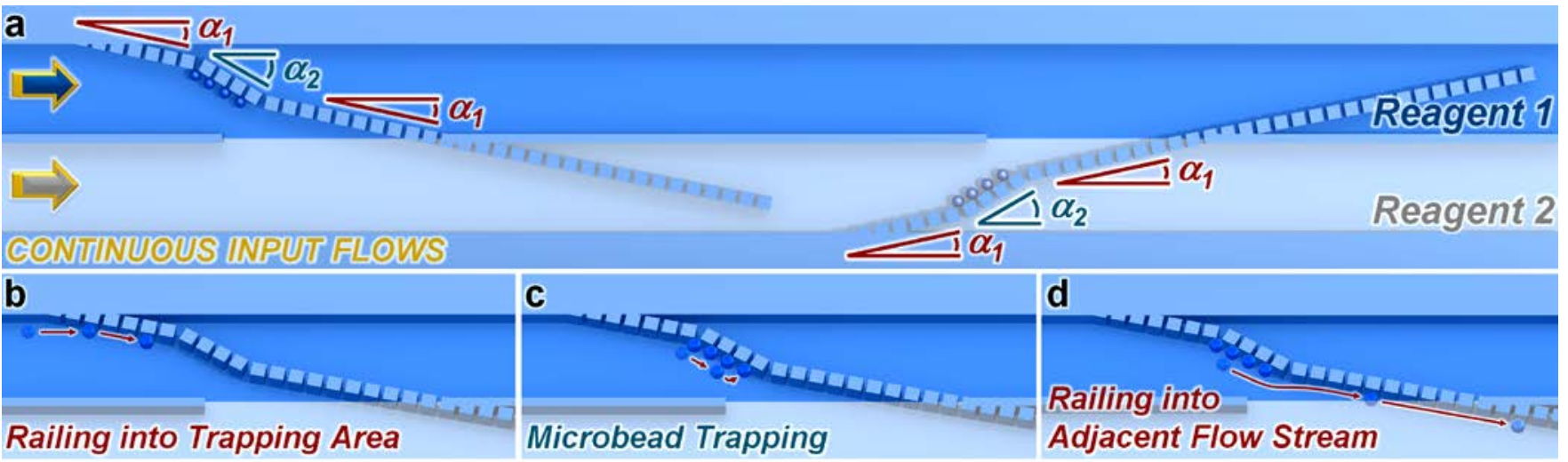

Figure 1: The microfluidic "rail-and-trap" concept. (a) Overview of an example system with two reagents inputted under continuous flow conditions. Microposts are arrayed in sections at either a low angle, " $\alpha_{1}$," or a higher angle, " $\alpha_{2}$," (with respect to the flow direction) in order to prevent or promote microbead immobilization, respectively. (b) Initially, suspended microbeads are railed into the trapping area due to the low $\alpha_{1}$. (c) The higher $\alpha_{2}$ promotes microbead trapping in the gaps between microposts. (d) After select numbers of microbeads are trapped, subsequent beads are railed into discrete, adjacent fluidic streams. This process can be repeated as desired (and additional reagents and/or washes can be loaded in parallel) in order to autonomously rail-and-trap microbeads during multi-stage mixing reactions. 

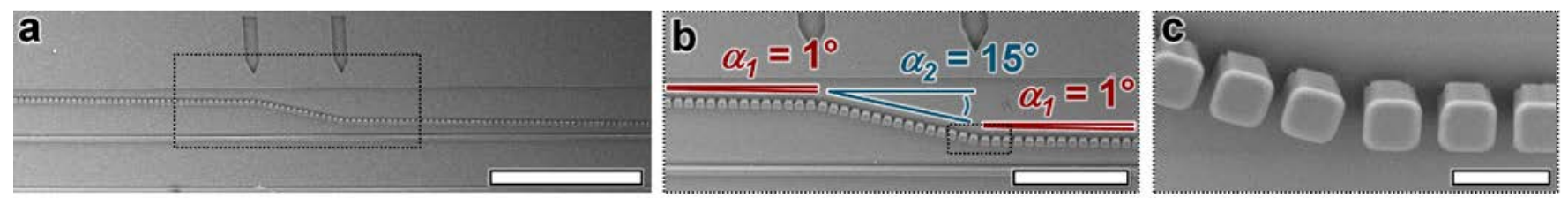

Figure 2: Microfabrication results for the microfluidic rail-and-trap system. Microposts are arrayed in sections at $\alpha_{1}=1{ }^{\circ}, \alpha_{2}=15^{\circ}$, and

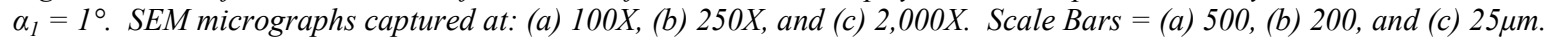

\section{CONCEPT}

The ability to visualize microbeads during specified points of multi-stage reaction processes is a critical requirement for a variety of biochemical assays. For example, we previously achieved beadbased cytokine detection via a multi-stage aptamer-based sandwich assay that consisted of three fundamental steps [22]. First, a fluorescent aptamer was conjugated to the microbead substrates, which resulted in the highest bead-based fluorescent intensities (i.e., for the positive control). Then, a complementary quencher was bound to the fluorescent aptamer, which quenched the fluorescent signal (i.e., for the negative control). Lastly, an inflammatory cytokine, IFN- $\gamma$, displaced the quencher by binding to the fluorescent aptamer, which resulted in a detectable increase in the fluorescent intensity on the microbead surfaces [22]. For such assays, the utility of our prior microfluidic railing system remains limited, despite the broad advantages associated with autonomous bead-based fluidic mixing on-chip [21].

To overcome this drawback, here we present a microfluidic "rail-and-trap" system (Fig. 1). Previously, we observed that square microposts arrayed at an angle of $1^{\circ}$ successfully railed microbeads by preventing microbead immobilization in the system; however, higher angles (e.g., 15 ${ }^{\circ}$ ) were found to promote microbead immobilization in the gaps between microposts [21]. In this work, we exploit these phenomena in order to passively: (i) transport suspended microbeads into distinct, parallel flow streams, and (ii) immobilize select numbers of microbeads during each phase of multi-stage fluidic processes. Figure 1a shows illustrations of the microfluidic rail-and-trap concept, which consists of square-shaped microposts (i.e., of approximately the same size as the microbeads) arrayed in sections at either a low angle, $\alpha_{1}$, or a higher angle, $\alpha_{2}$, in order to prevent or promote microbead immobilization, respectively. Under continuous flow conditions, microbeads are initially guided toward the trapping area due to the low $\alpha_{1}$ (Fig. 1b). The higher $\alpha_{2}$ promotes microbead immobilization in the gaps between microposts (Fig. 1c). After select numbers of microbeads are immobilized, subsequent microbeads are transported into the distinct, adjacent fluidic stream (Fig. 1d). This process can be repeated continuously as desired. Furthermore, additional fluidic reagents and/or washes can be inputted in parallel to tailor the microfluidic rail-and-trap system for a variety of multi-stage fluidic reaction processes.

\section{MICROFABRICATION}

The prototype rail-and-trap system was fabricated via standard and universally employed single-layer soft-lithography processes using polydimethylsiloxane (PDMS). Briefly, an $18 \mu \mathrm{m}-$ high layer of SU-8 negative photoresist was spin-coated onto a clean $\mathrm{Si}$ wafer. Using a photomask, the device patterns were UV exposed onto the layer of photoresist via contact photolithography. The wafer was then developed to become a positive master for the micromolding process. Next, the silicone elastomer, PDMS, was mixed at a 10:1 (base : curing agent) ratio and poured onto the master. After curing of the PDMS at $55{ }^{\circ} \mathrm{C}$, the elastomer was removed from the master. Individual devices were cut and then punched with holes at inlet and outlet locations. Glass slides were washed in successive dishes of Acetone, Isopropanol, and DI water, while the PDMS devices were washed in successive dishes of Isopropanol and DI water. The glass slides and PDMS devices were dried with a $\mathrm{N}_{2}$ gun, and then exposed to UV ozone for 5 minutes. Lastly, the PDMS devices were thermally bonded to the glass slides at $55{ }^{\circ} \mathrm{C}$. The final devices included arrays of square-shaped microposts $\left(15 \times 15 \mu \mathrm{m}^{2}\right)$, with an interpost spacing of $5 \mu \mathrm{m}$. The microchannels included a width of $200 \mu \mathrm{m}$. Microfabrication results are shown in Figure 2.

\section{RESULTS \\ COMSOL Multiphysics Fluid Dynamics Simulations}

Three-dimensional COMSOL Multiphysics fluid dynamics simulations were performed to provide insight into the microfluidic rail-and-trap system (Fig. 3). The model included spherical microbeads (15 $\mu \mathrm{m}$ in diameter) placed in contact with arrayed square microposts $\left(15 \times 15 \mu \mathrm{m}^{2}\right)$ in each section of the system (i.e., $\alpha_{1}=1^{\circ}, \alpha_{2}=15^{\circ}$, and $\alpha_{1}=1^{\circ}$ ). The pressure field simulation results showed higher pressure drops across the arrayed microposts in the $\alpha_{2}=15^{\circ}$ section compared to the $\alpha_{1}=1^{\circ}$ sections. Additionally, the fluid velocity field vectors (overlaid red arrows) located at the top right of the microbeads had opposite directionalities for the $\alpha_{1}=1^{\circ}$ sections versus the $\alpha_{2}=15^{\circ}$ section, with the velocity field vectors directed into the gap for the $\alpha_{2}=15^{\circ}$ case. These simulation results suggest that the $\alpha_{1}=1^{\circ}$ sections would promote railing of suspended microbeads along the arrayed microposts, while the $\alpha_{2}=15^{\circ}$ section would be more likely to promote microbead trapping in the gaps between microposts.

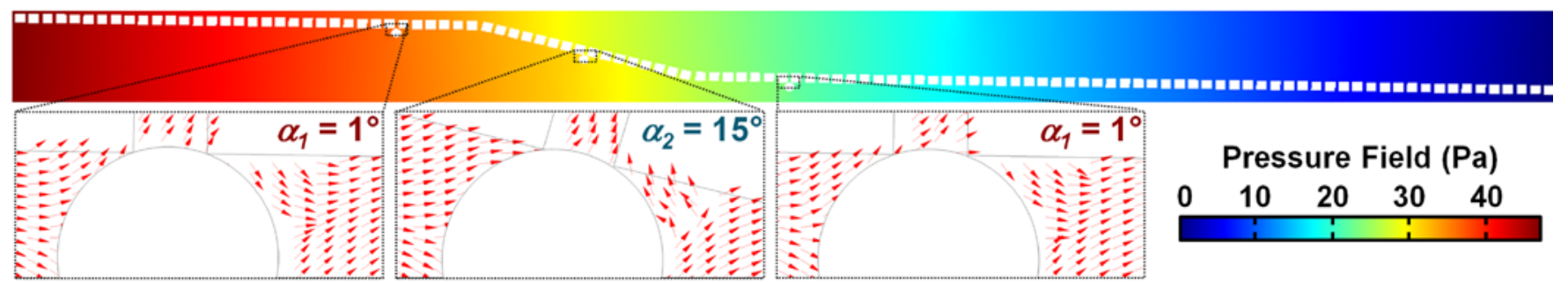

Figure 3: Three-dimensional COMSOL Multiphysics fluid dynamics simulation results for the microfluidic rail-and-trap system. The model consists of spherical microbeads $\left(15 \mu \mathrm{m}\right.$ in diameter) in contact with arrayed microposts $\left(15 \times 15 \mu \mathrm{m}^{2}\right)$ in each section of the system (i.e., $\alpha_{1}=1^{\circ}, \alpha_{2}=15^{\circ}$, and $\alpha_{1}=1^{\circ}$ ). (Top) Pressure field across the rail-and-trap system. (Bottom) Expanded views of each of the microbeads. Overlaid 'red arrows' denote the direction of the fluid velocity field vectors. 

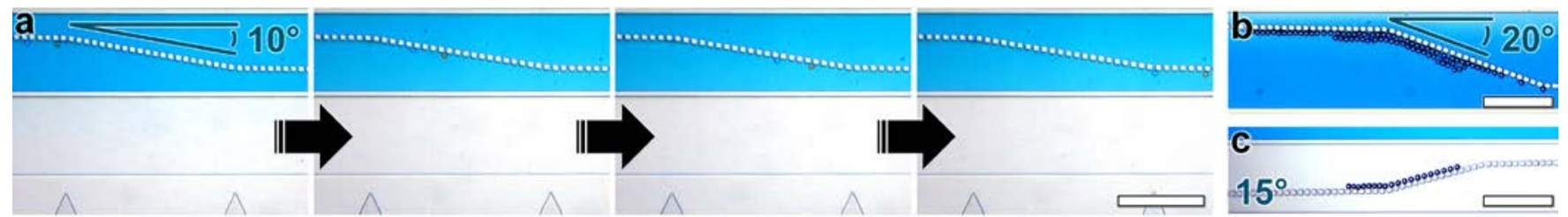

Figure 4: Experimental results for microbead handling in various microfluidic rail-and-trap testing systems. (a) Sequential micrographs of a suspended microbead bypassing the trapping area of a system with $\alpha_{1}=1^{\circ}$ and $\alpha_{2}=10^{\circ}$. (b) A micrograph of the trapping area of a system with $\alpha_{1}=1^{\circ}$ and $\alpha_{2}=20^{\circ}$ after the microbead loading process. (c) A micrograph of the trapping area of a system with $\alpha_{1}=1^{\circ}$ and $\alpha_{2}=15^{\circ}$ after the microbead loading process. Suspended streptavidin-coated polystyrene microbeads are $15 \mu \mathrm{m}$ in diameter; 'Orange' and 'blue' microbeads show a singular mobile microbead at two time-points within one second; Scale Bars $=200 \mu \mathrm{m}$.

\section{Experimental Characterization of Rail-and-Trap Performance}

Experiments with suspended streptavidin-coated polystyrene microbeads $(15 \mu \mathrm{m}$ in diameter) were performed using testing systems with varying $\alpha_{2}$ (i.e., while $\alpha_{1}$ was held constant at $1^{\circ}$ ) in order to investigate the effects of $\alpha_{2}$ on rail-and-trap performance. Previously, we found that values of $\alpha$ as low as $2.5^{\circ}$ resulted in microbead immobilization with the railing system, with higher proportions of microbead trapping observed as the value of $\alpha$ increased [21]. In this study, a variety of testing devices were fabricated with values of $\alpha_{2}$ ranging from $5^{\circ}$ to $25^{\circ}$ (with $\alpha_{1}=1^{\circ}$ ). A blue-dyed microbead suspension and a clear wash solution of phosphate buffered saline (PBS) were simultaneously loaded via separate inlets under continuous, constant input flow conditions. Specifically, two syringe pumps - each set at a constant flow rate of $1.5 \mu \mathrm{l} / \mathrm{min}$ - were used to control the input flow rates for each solution/suspension in parallel.

For testing systems in which $\alpha_{2}$ was too low (i.e., $\alpha_{2} \leq 10^{\circ}$ ), microbeads were observed to bypass the trapping areas without being immobilized. For example, Figure 4a shows sequential micrographs of a suspended microbead railing along the $\alpha_{2}=10^{\circ}$ trapping area, instead of being immobilized. Conversely, for systems where $\alpha_{2}$ was too high (i.e., $\alpha_{2} \geq 20^{\circ}$ ), microbead immobilization in the trapping area was promoted too strongly, which caused device failure because subsequent microbeads were prevented from bypassing fully occupied trapping areas. For example, Figure $4 \mathrm{~b}$ shows an $\alpha_{2}=20^{\circ}$ testing system after the bead loading process, with high numbers of microbeads immobilized on top of previously trapped microbeads. Experimental device runs revealed that the prototype systems with $\alpha_{2}=15^{\circ}$ were the most effective systems tested for trapping select numbers of microbeads without preventing subsequent microbeads from railing into the distinct, parallel flow streams (Fig. 4c).
Figure 5 shows experimental results for the microfluidic railand-trap system with $\alpha_{1}=1^{\circ}$ and $\alpha_{2}=15^{\circ}$. Under continuous input flow conditions, suspended microbeads were successfully: (a) railed into the $\alpha_{2}$ trapping area for immobilization, (b) transported along the micropost array rails from the top solution (blue) into the discrete, adjacent bottom solution (clear), (c) directed to bypass previously immobilized microbeads in the $\alpha_{2}$ trapping area, and $(d)$ transported along the micropost array rails from the bottom solution (clear) back into the parallel top solution (blue). Multiple experimental device runs confirmed that this process was repeatable. One caveat to this initial demonstration, however, was that select numbers of microbeads were found to immobilize in the $\alpha_{1}$ section directly preceding the $\alpha_{2}$ trapping areas (Fig. 4c; Fig. 5a, c). This phenomenon necessitated that higher numbers of microbeads be loaded initially in order to account for the expected loss of microbeads. Thus, one focus area for future research would be to adapt the current design to eliminate this issue. Despite this limitation, the overall device performance for the prototype system was not impacted significantly (Fig. 5). Rather, the majority of microbeads were still found to rail-and-trap as designed.

\section{CONCLUSIONS}

Microfluidic processors that are capable of autonomous onchip functionalities are critical to the advancement of lab-on-a-chip applications, such as point-of-care (POC) molecular diagnostics and biomarker screening. This work represents the first demonstration of a microfluidic system that functions autonomously under continuous input flow conditions to both transport suspended microbeads into distinct, parallel flow streams for fluidic mixing, while immobilizing select numbers of microbeads at each step of multi-stage fluidic processes as desired.

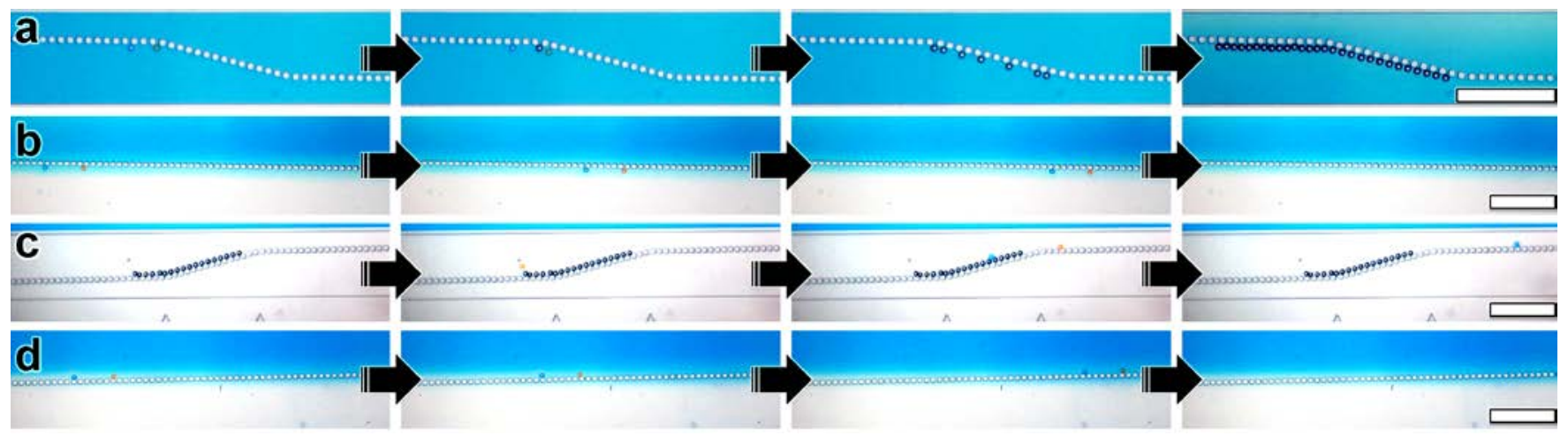

Figure 5: Sequential micrographs of experimental results for microbead dynamics in the microfluidic rail-and-trap system with $\alpha_{1}=1^{\circ}$ and $\alpha_{2}=15^{\circ}$. (a) Microbeads are railed into the trapping area and then immobilized. (b) A microbead is railed from the top solution (blue) into the bottom wash solution (clear). (c) A microbead bypasses previously immobilized microbeads. (d) A microbead is railed from the bottom wash solution (clear) back to the top solution (blue). Suspended streptavidin-coated polystyrene microbeads are 15 um in diameter; 'Orange' and 'blue' microbeads show a singular mobile microbead at two time-points within one second; Scale Bars $=200 \mu$ m. 
Specifically, microposts arrayed in sections at angles of $1^{\circ}, 15^{\circ}$, and $1^{\circ}$ (with respect to the flow direction) were utilized to: (i) guide suspended microbeads into a designated trapping area, (ii) immobilize select numbers of microbeads in the trapping area, and then (iii) rail subsequent microbeads into discrete, adjacent flow streams, respectively. Both theoretical simulations and experimental methods were employed to characterize the presented microfluidic rail-and-trap system. Experimental results revealed that trapping areas with microposts arrayed at lower angles (i.e., $\alpha_{2} \leq 10^{\circ}$ ) were unable to immobilize suspended microbeads within the device, while microposts arrayed at higher angles (i.e., $\alpha_{2} \geq 20^{\circ}$ ) resulted in device failure because subsequent microbeads immobilized on top of previously arrayed beads. In contrast, systems that included trapping areas with microposts arrayed at an angle of $15^{\circ}$ were found to rail-and-trap effectively. One limitation of the presented system was that a select number of microbeads were found to unintentionally immobilize in the railing section directly preceding fully occupied trapping areas. Although future work should focus on modifying the current system to overcome this issue, the overall device performance was not impacted significantly. Thus, the presented microfluidic rail-andtrap system provides a robust single-layer, continuous flow technique for autonomously accomplishing multiple fluidic mixing processes with suspended microbeads (e.g., for chemical and biological assays), with the unique capability of enabling microbead visualization and signal detection during each phase of multi-stage reactions.

\section{ACKNOWLEDGEMENTS}

The authors greatly appreciate the help and support of Kosuke Iwai, Adrienne Higa, and Paul Lum. The authors also thank the members of the Liwei Lin Laboratory, the Biologically-inspired Photonics-Optofluidics-Electronics Technology and Science (BioPOETS) Laboratory, and the Micro Mechanical Methods for Biology $\left(\mathrm{M}^{3} \mathrm{~B}\right)$ Laboratory. This work is supported in part by the DARPA N/MEMS program under the Micro/Nano Fluidics Fundamentals Focus (MF3) center.

\section{REFERENCES}

[1] P. Yager, T. Edwards, E. Fu, K. Helton, K. Nelson, M. R. Tam, and B. H. Weigl, "Microfluidic diagnostic technologies for global public health," Nature, 442, pp. 412-418, (2006).

[2] T. Thorsen, S. J. Maerkl, and S. R. Quake, "Microfluidic large-scale integration," Science, 298, pp. 580-584, (2002).

[3] A. D. Tadmor, E. A. Ottesen, J. R. Leadbetter, and R. Phillips, "Probing Individual Environmental Bacteria for Viruses by Using Microfluidic Digital PCR,” Science, 333, pp. 58-62, (2011).

[4] K. Iwai, W.-H. Tan, H. Ishihara, and S. Takeuchi, "A resettable dynamic microarray device," Biomedical Microdevices, (2011).

[5] W. H. Tan and S. Takeuchi, "A trap-and-release integrated microfluidic system for dynamic microarray applications," Proceedings of the National Academy of Sciences of the United States of America, 104, pp. 1146-1151, (2007).

[6] W. H. Tan and S. Takeuchi, "Dynamic microarray system with gentle retrieval mechanism for cell-encapsulating hydrogel beads," Lab on a Chip, 8, pp. 259-266, (2008).

[7] J. H. Wang, W. Wang, Y. H. Liu, L. B. Duo, L. J. Huang, and X. F. Jiang, "The method of single-nucleotide variations detection using capillary electrophoresis and molecular beacons," Molecular Biology Reports, 36, pp. 1903-1908, (2009).

[8] E. Verpoorte, "Beads and chips: new recipes for analysis,"
Lab on a Chip, 3, pp. 60N-68N, (2003).

[9] R. D. Sochol, B. P. Casavant, M. E. Dueck, L. P. Lee, and L. Lin, "A dynamic bead-based microarray for parallel DNA detection," Journal of Micromechanics and Microengineering, 21, p. 054019, (2011).

[10] N. Tuleuova, C. N. Jones, J. Yan, E. Ramanculov, Y. Yokobayashi, and A. Revzin, "Development of an Aptamer Beacon for Detection of Interferon-Gamma," Analytical Chemistry, 82, pp. 1851-1857, (2010).

[11] S. A. Dunbar, "Applications of Luminex (R) xMAP (TM) technology for rapid, high-throughput multiplexed nucleic acid detection," Clinica Chimica Acta, 363, pp. 71-82, (2006).

[12] F. J. Steemers and K. L. Gunderson, "Whole genome genotyping technologies on the BeadArray (TM) platform," Biotechnology Journal, 2, pp. 41-49, (2007).

[13] D. C. Leslie, C. J. Easley, E. Seker, J. M. Karlinsey, M. Utz, M. R. Begley, and J. P. Landers, "Frequency-specific flow control in microfluidic circuits with passive elastomeric features," Nature Physics, 5, pp. 231-235, (2009).

[14] B. Mosadegh, C.-H. Kuo, Y.-C. Tung, Y.-s. Torisawa, T. Bersano-Begey, H. Tavana, and S. Takayama, "Integrated elastomeric components for autonomous regulation of sequential and oscillatory flow switching in microfluidic devices," Nature Physics, 6, pp. 433-437, (2010).

[15] J. A. Weaver, J. Melin, D. Stark, S. R. Quake, and M. A. Horowitz, "Static control logic for microfluidic devices using pressure-gain valves," Nature Physics, 6, pp. 218-223, (2010).

[16] S. E. Chung, S. A. Lee, J. Kim, and S. Kwon, "Optofluidic encapsulation and manipulation of silicon microchips using image processing based optofluidic maskless lithography and railed microfluidics," Lab on a Chip, 9, pp. 2845-2850, (2009).

[17] S. E. Chung, W. Park, S. Shin, S. A. Lee, and S. Kwon, "Guided and fluidic self-assembly of microstructures using railed microfluidic channels," Nature Materials, 7, pp. 581587, (2008).

[18] P. Abbyad, R. Dangla, A. Alexandrou, and C. N. Baroud, "Rails and anchors: guiding and trapping droplet microreactors in two dimensions," Lab on a Chip, 11, pp. 813-821, (2011).

[19] C. Kantak, S. Beyer, L. Yobas, T. Bansal, and D. Trau, “A 'microfluidic pinball' for on-chip generation of Layer-byLayer polyelectrolyte microcapsules," Lab on a Chip, 11, pp. 1030-1035, (2011).

[20] S. Zhang, L. Yobas, and D. Trau, "A Microfluidic Device for Continuous Flow Layer-by-Layer Encapsulation of Droplets with Polyelectrolytes," presented at The 12th International Conference on Miniaturized Systems for Chemistry and Life Sciences (MicroTAS 2008), San Diego, CA, USA, (2008), pp. 1402-1404.

[21] R. D. Sochol, R. Ruelos, V. Chang, M. E. Dueck, L. P. Lee, and L. Liwei, "Continuous flow layer-by-layer microbead functionalization via a micropost array railing system," presented at The 16th International Solid-State Sensors, Actuators and Microsystems Conference (Transducers 2011), (2011).

[22] R. D. Sochol, L. Lo, R. Ruelos, V. Chang, D. Bahri, K. Iwai, J. C. Lo, M. E. Dueck, L. P. Lee, and L. Lin, "Microfluidic Ping Pong for Cytokine Detection," presented at The IEEE 24th International Conference on Micro Electro Mechanical Systems (MEMS 2011), Cancun, Mexico, (2011).

\section{CONTACT}

*Ryan D. Sochol, tel: +1-410-935-8971; rsochol@gmail.com 